\title{
Parallel search for maximum satisfiability
}

\author{
Ruben Martins ${ }^{1}$
}

Published online: 10 September 2015

(C) Springer Science+Business Media New York 2015

\begin{abstract}
The predominance of multicore processors has increased the interest in developing parallel Boolean Satisfiability (SAT) solvers. As a result, more parallel SAT solvers are emerging. Even though parallel approaches are known to boost performance, parallel solvers developed for Boolean optimization are scarce.
\end{abstract}

This dissertation proposes parallel search algorithms for Maximum Satisfiability (MaxSAT) and introduces PWBO, the first parallel solver for MaxSAT. PWBO can use two different strategies for parallel search. The first strategy performs a portfolio approach by searching on the lower and upper bound values of the optimal solution using different encodings of cardinality constraints for each thread. The second strategy splits the search space considering different upper bound values of the optimal solution for each thread.

As others parallel solvers, PWBO suffers from non-deterministic behavior, i.e. several runs of the same solver can lead to different solutions. This is a clear downside for applications that require solving the same problem instance more than once. Therefore, we also present the first deterministic parallel MaxSAT solver that ensures reproducibility of results. Finally, we also propose partitioning techniques to improve sequential MaxSAT algorithms.

School: Instituto Superior Técnico, Universidade de Lisboa, Portugal

\section{Supervisors:}

Inês Lynce

Vasco Manquinho

Graduated: Monday, September 9, 2013

Ruben Martins

rmartins@utexas.edu 
Link to full text: http:/www.a4cp.org/sites/default/files/ruben_martins_-_parallel_search_ for_maximum_satisfiability.pdf

Also published in:

Community-based Partitioning for MaxSAT Solving

Parallel Search for Maximum Satisfiability

An Overview of Parallel SAT Solving

Deterministic Parallel MaxSAT Solving

On Partitioning for Maximum Satisfiability

Clause Sharing in Parallel MaxSAT

Exploiting Cardinality Encodings in Parallel Maximum Satisfiability

Improving Search Space Splitting for Parallel SAT Solving

\section{Notes:}

Concluded the $\mathrm{PhD}$ degree with the grade "Passed with Distinction", which is only awarded to less than $10 \%$ of the $\mathrm{PhD}$ students at IST.

Awards for solvers developed during $\mathrm{PhD}$ :

1 bronze medal at PB Evaluation 2011

2 silver medals and 1 bronze medal at MaxSAT Evaluation 2011

2 gold medals, 1 silver medal and 1 bronze medal at MaxSAT Evaluation 2012 